\title{
Video Article \\ The Knob Supination Task: A Semi-automated Method for Assessing Forelimb Function in Rats
}

\author{
Samuel D. Butensky ${ }^{1}$, Thelma Bethea ${ }^{1}$, Joshua Santos ${ }^{1}$, Anil Sindhurakar ${ }^{1}$, Eric Meyers ${ }^{2,3}$, Andrew M. Sloan ${ }^{2,3}$, Robert L. Rennaker $I^{2,3}$, Jason \\ B. Carmel ${ }^{1,4,5}$ \\ ${ }^{1}$ Burke Medical Research Institute \\ ${ }^{2}$ Texas Biomedical Center, The University of Texas at Dallas \\ ${ }^{3}$ Erik Jonsson School of Engineering and Computer Science, The University of Texas at Dallas \\ ${ }^{4}$ Brain and Mind Research Institute, Weill Cornell Medical College \\ ${ }^{5}$ Departments of Neurology and Pediatrics, Weill Cornell Medical College
}

Correspondence to: Jason B. Carmel at jason.carmel@med.cornell.edu

URL: https://www.jove.com/video/56341

DOI: doi:10.3791/56341

Keywords: Behavior, Issue 127, Dexterity, Forelimb, Supination, Automated, Behavior, Reaching, Corticospinal

Date Published: 9/28/2017

Citation: Butensky, S.D., Bethea, T., Santos, J., Sindhurakar, A., Meyers, E., Sloan, A.M., Rennaker II, R.L., Carmel, J.B. The Knob Supination Task: A Semi-automated Method for Assessing Forelimb Function in Rats. J. Vis. Exp. (127), e56341, doi:10.3791/56341 (2017).

\section{Abstract}

Tasks that accurately measure dexterity in animal models are critical to understand hand function. Current rat behavioral tasks that measure dexterity largely use video analysis of reaching or food manipulation. While these tasks are easy to implement and are robust across disease models, they are subjective and laborious for the experimenter. Automating traditional tasks or creating new automated tasks can make the tasks more efficient, objective, and quantitative. Since rats are less dexterous than primates, central nervous system (CNS) injury produces more subtle deficits in dexterity, however, supination is highly affected in rodents and crucial to hand function in primates. Therefore, we designed a semi-automated task that measures forelimb supination in rats. Rats are trained to reach and grasp a knob-shaped manipulandum and turn the manipulandum in supination to receive a reward. Rats can acquire the skill within $20 \pm 5$ days. While the early part of training is highly supervised, much of the training is done without direct supervision. The task reliably and reproducibly captures subtle deficits after injury and shows functional recovery that accurately reflects clinical recovery curves. Analysis of data is performed by specialized software through a graphical user interface that is designed to be intuitive. We also give solutions to common problems encountered during training, and show that minor corrections to behavior early in training produce reliable acquisition of supination. Thus, the knob supination task provides efficient and quantitative evaluation of a critical movement for dexterity in rats.

\section{Video Link}

The video component of this article can be found at https://www.jove.com/video/56341/

\section{Introduction}

A loss of dexterity after nervous system injury or disease significantly diminishes independence and quality of life for affected individuals ${ }^{1,2,3,4}$ Thus, dexterity is an important outcome measure for understanding the science of neural repair and rehabilitation as well the foundations of neural control of movement and motor learning. Traditionally, manual tasks like single pellet reaching, pasta manipulation, and Irvine, Beatties, and Bresnahan (IBB) Forelimb Scale have been used to evaluate dexterity in animals, specifically rodents ${ }^{5,6,7}$. These tasks have become popularized due to their minimal task acquisition time. However, they are qualitative in nature, laborious for the experimenter, and, at times, insensitive to functional impairment after injury with subtle deficits ${ }^{5,7,8,9}$. These limitations of traditional tasks have spurred the development of more quantitative measures of motor function in animals, specifically, forelimb reaching.

There are several advantages to automating tasks, namely objectivity, increased throughput, and decreased analysis time. New automated tasks provide a more sensitive measure of evaluating dexterity after injury than conventional tasks ${ }^{8,10}$. In addition, they allow for adaptive training and testing which tailors the training and testing difficulty to an animal's performance. Lastly, automated tasks generate large amounts of data, which provide two advantages. Firstly, an increase in data both within a trial and in the number of trials increases the statistical power of a study. Secondly, it gives neuroscientists a larger data set from which to study motor learning, training, and compensation more robustly through analysis of kinetic and kinematic information ${ }^{11}$.

Several groups have attempted to automate traditional tasks. High speed cameras can be used to gather kinematic data from tasks like the single pellet reaching task ${ }^{12}$. Alaverdashvili and Wishaw have used high-speed cameras to capture reaching movements and analyze digit movements using frame-by-frame motion measurement software Peak Motus ${ }^{13}$. However, this software does not identify digits using computer vision, but instead requires the experimenter to digitize moving points by cursor. Additionally, some tasks have been used in conjunction with feeders and cages to automate the training process ${ }^{14,15,16}$. 
Other groups have used force sensors as well as high speed cameras to evaluate spatial adjustments and force in skilled forelimb reaching using pasta manipulation, while others have designed tasks to capture more complex movements ${ }^{17}$. One such task is a reach and pull task that uses a three-degree-of-freedom robotic device to capture planar and rotational movement of rat forelimb movements ${ }^{18}$. This has advantages in being able to measure the kinetics of movements but with an increase in complexity and cost.

Here, we demonstrate a semi-automated forelimb task that measures supination in rats ${ }^{8}$. Forelimb supination is the rotation of the paw from palm down to palm up. Supination is both an excellent marker of corticospinal tract function and a clinically relevant movement in humans that is required for daily living activities ${ }^{8,19,20}$. In addition, supination is highly sensitive to injury and inactivation, especially when compared to single pellet reaching ${ }^{8}$. The supination task, developed in a collaboration between the Burke Medical Research Institute and The University of Texas at Dallas, measures rotational movement in the horizontal plane ${ }^{8,10}$. Rats are placed in a behavioral box (Figure 1A) and are trained to do three movements (Figure 1B): reach through a rectangular aperture; grasp a spherical manipulandum; supinate to a designated angle.

The behavior task is controlled by PC software (Figure 1C). The controlling software sends instructions to a microcontroller that's connected to the auto-positioner, optical encoder, speaker, and feeder. The microcontroller and its peripheral connections are referred to as the microcontroller box. Information flows from optical encoder, to the microcontroller, then the computer, and then back to the microcontroller. If the controlling software has signaled to the microcontroller that the trial was a success, the microcontroller triggers the feeder to dispense a pellet. At the beginning of each session, the controlling software relays the stage information to the microcontroller, which directs the auto-positioner to position the knob at the stage's defined distance from the aperture. The auto-positioner can also be operated manually by using the arrow keys located on the auto-positioner. The optical encoder records data at $100 \mathrm{~Hz}$ and measures changes in angle. All the data are stored in binary format.

The experimenter uses sequential training stages within the software to train the rat from habituation to supinating at a predetermined angle and success rate. During habituation, the knob manipulandum is placed inside the aperture window without any counterweight. After a week of highly supervised training, the rat associates the knob with a reward and begins turning the knob independently. Once the rat is able to turn independently, the knob is retracted to $1.25 \mathrm{~cm}$ in $0.25 \mathrm{~cm}$ increments until the rat can turn independently at $1.25 \mathrm{~cm}$. Counterweight is then added in $1 \mathrm{~g}$ increments from $3 \mathrm{~g}$ to $6 \mathrm{~g}$. Automated training stages train the animal to supinate the knob at $6 \mathrm{~g}$ up to 75 degrees. This stage of training is largely unsupervised; once rats adopt the task with proper form (discussed below), they continue to supinate properly. Training is complete when rats supinate 75 degrees at a success rate (hit rate) of $75 \%^{8}$. Here, we describe a typical training protocol and present solutions to common issues we have encountered. We demonstrate the progression of representative successful and unsuccessful rats through the training protocol, and show that the task can be modified to show functional impairment with subtle or more severe deficits.

\section{Protocol}

This protocol describes setting up the task and establishing living and feeding conditions, as well as training animals, testing animals after injury, and analyzing behavior data. The four steps of animal training are described as well: habituation, reward association, counterweight training, and training to criterion turn angle. All animal experiments were approved by the IACUC of Weill Cornell Medicine and University of Texas at Dallas.

\section{Setting Up the Task}

1. Design a behavioral box made of clear plastic that measures $150 \mathrm{~mm}$ wide by $200 \mathrm{~mm}$ long by $250 \mathrm{~mm}$ tall. NOTE: Here, the rectangular aperture is $14.2 \mathrm{~mm}$ wide by $25.4 \mathrm{~mm}$ high. The knob manipulandum of diameter $9.5 \mathrm{~mm}$ is made with methacrylate and has two stops that restrict supination to less than $100^{\circ}$. Each of these parameters have been tested and optimized for the deficits described in the results section. The behavioral box and manipulandum are shown in Figure 1A.

2. Connect the microcontroller box to the computer. Up to four microcontroller boxes can be connected to each computer.

3. To begin the task, use the control software (Figure 1C).

1. Select the knob device for control. Input the subject name in the "Subject" field. Choose the stage for training under the "Stage" dropdown menu.

NOTE: The stages dictates three parameters for each trial: the "Hit Window," "Hit Threshold," and "Initial Threshold." A hit is defined as reaching the criterion angle within a defined period of time. "Hit Window" refers to the time allotted for an animal to start a trial and reach the criterion angle. "Hit Threshold" is the criterion angle for a successful trial. "Initial Threshold" is the criterion angle to initiate a trial and hit window; this indicates the start of the turn. More information on Stages can be seen below under the section "Training Animals."

2. Click "Start" to begin the training session. Click "Feed" to manually feed the animal and "Stop" to stop the training session. NOTE: Manual feeding should be used to maintain interest in the task and is described in detail in section 3 . After stopping the session, session data will be saved to the C: drive.

\section{Living and Feeding Conditions}

1. Use adult female Sprague Dawley rats (175-200 g). House rats together in standard cages with reversed $12 \mathrm{~h}$ dark/12 $\mathrm{h}$ light cycle. Perform all training and testing during the dark cycle with the lights dimmed.

NOTE: Female rats are used since they are easier to train ${ }^{21}$. Here Sprague-Dawley were used because it is a common strain used in motor training, and it is the predominant strain for transgenics. However, given the adjustable nature of the task (counterweight, turn angle criterion, distance to knob, and knob itself) these could easily be adjusted for different strains and larger (e.g. males) or smaller (e.g. Wistar) rats.

2. For the first 5 days of the study, give rats a full trough in addition to nutritionally complete, chocolate flavored pellets for five days. After the fifth day, food-restrict rats to $85 \%$ of their normal weight curve. 
3. After food restriction has begun, feed the rats $7.5 \mathrm{~g}$ of chocolate pellets (adjust up or down to maintain $85 \%$ of age-adjusted weight) throughout the entirety of their training. If rats have not received $7.5 \mathrm{~g}$ of chocolate pellets in their training sessions, supplement withstandard chow after the final session.

NOTE: Weekend feeding is ad libitum with standard chow until Sunday evening, then food-deprive overnight. When performing surgeries or other invasive interventions, allow food ad libitum at least three days before and then for three days after the procedure (depending on recovery times) before putting rats on food restriction again.

\section{Training Procedure}

NOTE: An overview of the training procedure is shown in Figure 3A. Throughout the protocol, train the rats twice daily, once in the morning and once in the afternoon. Wait at least $3 \mathrm{~h}$ after the morning session before starting the afternoon session.

\section{Habituation}

Note: The goal of habituation is to familiarize the rat with the testing box and with handling.

1. Retract the knob device fully by pressing the down arrow key on the auto positioner.

2. Place the rat in the behavioral box for $15 \mathrm{~min}$.

3. After $15 \mathrm{~min}$ in the box, handle each rat in the hands for at least five min to familiarize the rat to the experimenter.

4. Repeat this for five days.

\section{Reward Association}

NOTE: The goal is to train the rat to associate turning of the knob with a food reward. Expect to spend the full session length of 30 min on the task during the reward association stage of training.

1. Open the software and input the rat Name. Set the Stage to "K1: Knob Shaping - No Pulley."

NOTE: This sets the manipulandum distance away from the aperture at $0.0 \mathrm{~cm}$, the "Init. Thresh." at $3^{\circ}$, "HIT Thresh." at 5 degrees, and "HIT Window" at $2 \mathrm{~s}$. This allows the rat to be fed as long as the rat turns the knob past 3 degrees. The "HIT Window" will remain at $2 \mathrm{~s}$ throughout training.

2. Put the rat in the behavioral box and press "Start" to begin the session.

3. Familiarize the rat to the reward trough by dispensing $2-3$ pellets at a time and tapping the side of the box where the reward trough is located. Dispense the pellets by using the manual feeder button.

4. Once the rat knows the location of the reward, manually dispense 1 pellet when the rat is in front of the aperture.

5. Once the rat moves to the aperture in anticipation of a pellet reward, condition the rat to interact with the manipulandum.

1. Prompt the rat to engage by placing a $45 \mathrm{mg}$ food pellet near the manipulandum, or by applying pellet dust directly to the manipulandum, allowing it to reach out and grasp it. If the rat moves away from the aperture, tap on the box near the manipulandum to redirect its attention.

NOTE: If the rat has touched the knob with its reaching paw, the program will feed the rat. Any interactions that do not include the use of paws (i.e. biting, nosing the manipulandum) are incorrect and should not be rewarded.

6. Once the rat begins touching the knob with the desired paw and getting rewarded 10 consecutive times at $0.0 \mathrm{~cm}$, use the down arrow key on the auto-positioner (Figure 1A) to retract the manipulandum by $0.25 \mathrm{~cm}$. Repeat this in $0.25 \mathrm{~cm}$ increments until the manipulandum is $1.25 \mathrm{~cm}$ away from the aperture.

7. Press "Stop" after 30 min to end the session.

8. Continue reward association until the rat supinates the manipulandum with the desired paw at $1.25 \mathrm{~cm}$ away from the aperture (Figure 1B).

9. Once the rat has completed 2 consecutive sessions supinating the manipulandum and retrieving the pellet reward, begin counterweight training.

3. Counterweight training to train the rats to supinate a $6 \mathrm{~g}$ counterweight.

1. Place a $3 \mathrm{~g}$ counterweight on the manipulandum by attaching the connector at the end of the counterweight to the L-shaped attachment point on the manipulandum. Feed the counterweight string through the pulley until the counterweight hangs freely.

2. Open software and input the rat Name. Set the Stage to "K2: Knob Shaping - Pulley."

NOTE: This sets the manipulandum distance away from the aperture at $1.25 \mathrm{~cm}$, the "Init. Thresh." at $3^{\circ}$, and the "HIT Thresh." at 5 degrees. The manipulandum distance will remain at $1.25 \mathrm{~cm}$ from this point forward.

3. Put the rat in the behavioral box and press "Start" to begin the session.

4. Press "Stop" after $100+$ successful trials. Once the rat has completed 2 consecutive sessions of $100+$ successful trials, increase the weight by $1 \mathrm{~g}$ after the end of a session. Increase the weight from $3 \mathrm{~g}$ to $6 \mathrm{~g}$, over subsequent sessions.

5. Proceed to step 3.4 after 2 consecutive sessions of supination at $6 \mathrm{~g}$ and $100+$ successful trials.

NOTE: It takes an average of 6 sessions ( 3 days) to work up from $3 \mathrm{~g}$ to $6 \mathrm{~g}$. Beginning in counterweight training, it is imperative that correct supination movement gets rewarded and incorrect supination movement gets shaped out. For a visual guide and explanation about correct and incorrect supination form, please refer to Figure 2.

\section{Training to Baseline}

NOTE: Remember to continue to shape correct supination movement. Again, refer to Figure 2 for a visual guide on correct/incorrect supination movement and how to ameliorate incorrect supination. Train the rats to supinate to the baseline criteria; here, this is 75 degrees with a $6 \mathrm{~g}$ counterweight at a success rate of $75 \%$ or higher.

1. Place a $6 \mathrm{~g}$ counterweight on the manipulandum.

2. Open software and input the rat Name. Set the Stage to "KSB4: Knob Training Median 75 Max." NOTE: This sets the "Init. Thresh." at $5^{\circ}$, "HIT Threshold Minimum" at $15^{\circ}$, and "HIT Threshold Maximum" at $75^{\circ}$. This is called an "adaptive" training stage, meaning the threshold will increase as the rat's performance improves. For the "KSB4" stage, the threshold is first set to the previous session's final threshold. If no previous session was run on this stage, the threshold is set to the threshold 
minimum of 15 degrees. After the first 10 trials of the session, the threshold is calculated as the median of the peak angles in the previous 10 trials. Thus, the threshold is different for each trial and depends on the rat's performance in the previous trials.

3. Stop the session after $30 \mathrm{~min}$.

4. Continue training using the adaptive stage until the average peak angle is 75 degrees or greater. Then, proceed to step 3.5. This generally occurs after about 10 days or 20 sessions.

\section{Baseline assessment to record four consecutive baselines}

1. Place a $6 \mathrm{~g}$ counterweight on the manipulandum.

2. Open software and input the rat Name. Set the Stage to "K27: 75 degrees." This sets the "Init. Thresh." at $5^{\circ}$ and "HIT Threshold at $75^{\circ}$.

3. Press "Stop" after 30 min or 100 trials, whichever comes first, to end the session.

4. Continue until there are four consecutive baselines with a success rate of $75 \%$ or higher.

\section{Post-Injury Assessment}

1. Open software and input the rat name. Set the Stage to the same static stage as pre-injury baseline testing. This will retain the same parameters used for baseline.

2. Press "Start" and run session until $30 \mathrm{~min}$.

NOTE: Participation might be low after injury. This can be remedied by using the manual feed button or placing a reward pellet near the manipulandum to entice the rat to engage with the manipulandum.

3. Repeat testing once a week in weekly intervals until the desired length of post-injury assessment has been reached. NOTE: Here, we assess post-injury performance each week for six weeks.

\section{Analyze Data}

NOTE: Data are saved to a default location on the C: Drive of a PC. Data are captured at $100 \mathrm{~Hz}$ during the hit window and stored in binary format. Here, the data was analyzed using a custom program, called Dexterity. Please email Dr. Jason Carmel for access to this free software.

1. Open Dexterity and click "Standard."

2. Locate the directory where rat data is and select the desired folder.

3. After selecting the directory, select the rats for analysis.

4. Click "Keep" or "Discard" to keep or discard files that are incomplete or contain no data.

NOTE: There are times when sessions are started using the wrong parameters and training stage and while they are immediately stopped, a file is still created. This file needs to be discarded during analysis. There are also instances, especially acutely after injury, when an animal performs no trials because of their injury or very little trials. For these sessions, a file must be kept because it is a representation of their performance. When a file with no successful trials is kept, a NaN is placed in the appropriate place for computed variables.

5. Choose whether to annotate the experiment now or later; if "Annotate Now" is selected, a new window will open. Type in the "Experiment Name," "Group Names," "Event Data," and "Total Number of Weeks" in the experiment.

6. Assign subjects to a group.

7. Input the number of data sessions in each week or time point. Separate the input sessions by a space and make sure not to put a space after the last number i.e. (1 1111 1).

8. Input the time point labels. Separate input labels by a space and make sure not to put a space after the last label i.e. (Pre Wk1 Wk2 Wk3).

9. Select when the event occurred; an option to save the analysis session will appear. Click "Yes" to save the analysis session.

10. An overview of the annotated and unannotated data will appear. This gives the option to plot data.

11. For the unannotated data, click "Graph Subject" to plot an individual subject or "Graph Subjects" to plot all subjects on the same graph.

12. For the annotated analysis, click "Plot" to plot the experiment.

13. For both annotated and unannotated, click the dropdown arrow for a list of variables to be plotted. Click the variable to view the plot.

14. Click export to export the graph and/or the data associated with the graph.

\section{Representative Results}

Early in training, the experimenter spends more time on task shaping rat behavior. As the rats associate rat supination with reward, the hands on time decreases (Figure 3A). During habituation, reward association, and counterweight training, the full session length (30 min) is spent on the task. However, after a rat is supinating with a $6 \mathrm{~g}$ weight, the time on task gradually lessens to about 15 min as the rat's supination angle increases. Finally, when the rat reaches baseline, the time on task is at a minimum; the experimenter only needs to place the rat in the behavioral box and start the program. The maximum number of rats an experimenter can work with simultaneously is two rats during reward association, four rats during counterweight training and training to baseline, and as many rats as there are boxes during baseline assessment and post-injury testing. On average, $75 \%$ of rats $(n=56)$ acquire the task.

After the rat has associated supination with a reward, there is a positive progression in the rat's supination angle (Figure 3B). In Figure 3B, the rat progressed from $3 \mathrm{~g}$ to $6 \mathrm{~g}$ counterweight from day 3 to day 7 . After counterweight training, there was a short period of adaptive training from day 7 to day 9 , during which supination increased from 26 to 30 degrees. Because there was not much change, a static threshold was employed from day 9 to 18. During this period, the rat steadily increased from 30 degrees to 75 degrees in 8 days. There is day-to-day variability throughout training, in particular, days 12 and 14 . But generally, there is an upward trend in supination angle. By the end of day 17 posthabituation, the rat had recorded its first baseline, and four sessions later, it finished baseline assessment. From habituation to recording of a fourth baseline, the training protocol takes an average of $20 \pm 5$ days. 
While viewing an ideal progression through the training protocol is important, viewing an unsuccessful progression is equally important (Figure 4). In Figure 4A, the orange line shows a rat that successfully completes the protocol, the blue line shows an unsuccessful rat, and grey lines show another six successful rats. Successful rats reached baseline in $15 \pm 0.6$ days $(n=7)$. The representative successful rat uses a 1 o'clock grasp, while the unsuccessful rat uses a 3 o'clock grasp. Both rats associate the knob with a reward in 2 days. In addition, both rats show a similar supination angle (Figure 4A) progression in the first four days after the counterweight is added. However, after this point, the successful rat begins to break away from the unsuccessful rat. This is because the unsuccessful rat's grasp was unable to be corrected before this point (see Figure 2).

For the successful rat, there is a steep rise in supination angle that begins to plateau between 50 and 60 degrees but then resumes a steadier climb towards 75 degrees. However, for the unsuccessful rat, there is a more gradual increase in supination angle. As the rat plateaus around 20 degrees, the rat gets pushed to supinate more, but eventually, it loses interest in the task, even with manual feeding, and the supination angle decreases rapidly around Day 15 post-habituation. While there is a slight recovery after Day 17 post-habituation, the rat struggles to supinate more than 25 degrees. If a rat hasn't achieved baseline by day 20 , we consider this rat unsuccessful and remove the rat from the study.

In addition to supination angle, one can perform a visual inspection of the supination waveforms (Figure 4B-D) for the successful and unsuccessful rat. When performing a visual inspection, we look for several waveform characteristics: slope of the line, latency, and number of peaks in the time window for the trial. The slope of the line is calculated as the derivative of the curve between the onset of the curve and the peak of the curve. The latency is calculated as the time between the initiation of the trial and the curve crossing the hit threshold. Lastly, peaks are calculated by using the derivative to find local maxima in the trial window. Previously, we found that slope of the line, or velocity, is a robust measure of supination kinetics and is sensitive to subtle deficit ${ }^{8}$.

In the first third of training after beginning to supinate using $6 \mathrm{~g}$ (Figure 4B), the successful rat (Figure 4B1) shows a single waveform with a peak near 20 degrees, while the unsuccessful rat (Figure 4B2) shows a double turn, or two peaks, with the first peak near 10 degrees and the second peak near 5 degrees. In the middle third of training (Figure 4C), the successful rat (Figure 4C1) shows an increase in peak angle from 20 degrees to 50 degrees with a more defined, single peak curve. The unsuccessful rat (Figure 4C2), meanwhile, only shows a marginal increase in peak angle to 20 degrees but has improved in its form; it now only uses a single turn. By the final third of training (Figure 4D), the successful rat (Figure 4D1) shows a very pronounced single waveform with a peak around $65^{\circ}$, versus the unsuccessful rat (Figure 4D2) with a peak angle of 20 degrees but now with an additional peak at $2 \mathrm{~s}$ of $15^{\circ}$. This is another good indicator that with increasing training difficulty, the rat was unable to correct its 3 o'clock grasp, and in turn, unable to supinate properly. Even if this rat wasn't excluded from the study and could eventually perform up to 75 degrees, questions would remain about whether it was true supination versus supination with compensation.

Lastly, the supination task detects functional impairment after multiple types of injury, including a cut lesion of the corticospinal tract, the main pathway for voluntary movement in people, and forelimb motor cortex lesion performed with endothelin injections (Figure 5) ${ }^{\mathbf{8 1 0 , 2 2}}$. Rats in the pyramidotomy group (purple, $\mathrm{n}=8$ ) were trained to supinate at least $75^{\circ}$ at $6 \mathrm{~g}$ at a success rate of $75 \%$ or above, while rats in the cortical lesion group (green, $n=10$ ) were trained to supinate $60^{\circ}$ at $7.5 \mathrm{~g}$ at $75 \%$ or above. Rats in both groups showed a sharp decrease in success rate after injury (Figure 5A). Success rate for rats in the pyramidotomy group decreased from $90 \% \pm 2 \%$ to $14 \% \pm 8 \%$. The success rate for rats with cortical lesion decreased from $76 \% \pm 1 \%$ to $10 \% \pm 3 \%$. By week 6 , both groups were still impaired: the pyramidotomy group was at $34 \% \pm 11 \%$ while the cortical lesion group remained at $16 \% \pm 7 \%$. As for supination angle, both groups show a decrease from pre- to post-injury (Figure $5 \mathrm{~B}$ ). Due to the different criterion baseline supination angles, the pyramidotomy group had a higher pre-injury supination angle $\left(85^{\circ} \pm 2.9^{\circ}\right)$ than the cortical lesion group $\left(67^{\circ} \pm 0.52^{\circ}\right)$. The pyramidotomy group decreased to $38^{\circ} \pm 10^{\circ}$ while the cortical lesion group decreased to $27^{\circ} \pm 2.9^{\circ}$. 


\section{A. Supination Device}

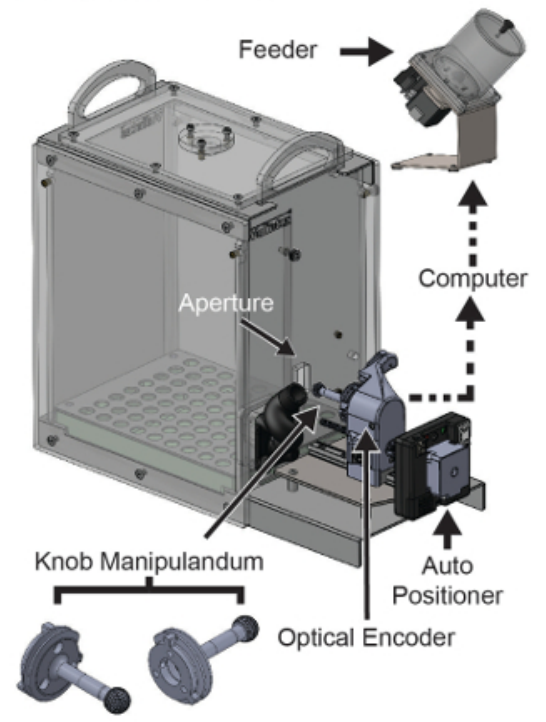

B. Rat Performing Task

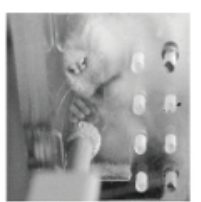

Reach

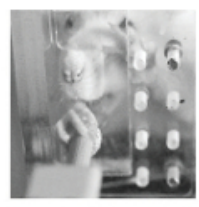

Grasp

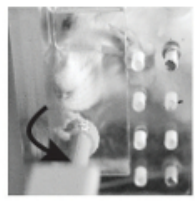

Supinate

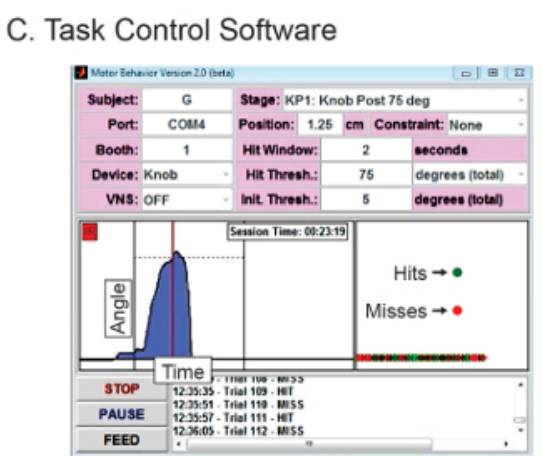

Figure 1: Supination Task Description. (A) The rat is placed in a Plexiglas box with an aperture through which it reaches and grasps a knob that must be turned in supination. The knob has two stops to prevent supination angles greater than $100^{\circ}$. The knob also has a pulley with counterweight; this creates torque that the rat must overcome to supinate. The knob is connected to an optical encoder that measures angle with an accuracy of $0.25^{\circ}$. This optical encoder is connected to a microcontroller, which in turn is connected to a computer which controls the task. The computer signals to the microcontroller when to trigger audio-feedback and dispense a pellet from the feeder if a success criterion is achieved. The microcontroller also controls the auto positioner whose position between 0 and $1.25 \mathrm{~cm}$ is dictated by the training stage set by the computer. (B) The rat performs the task in three successive movements: reaching through the aperture, grasping the knob with a power grasp located at 1 o'clock, and supinating. (C) The knob supination task is controlled by control software. The experimenter inputs the subject's name and chooses the training stage, while the program sets the corresponding parameters. A waveform of a single successful supination trial is shown in blue, while the sequence of successful and failed trials are shown in green and red, respectively. A trial is marked successful by the control software if the supination angle is greater than the hit threshold within the defined time window, whereas a trial is marked unsuccessful if it does not. This program controls one box. Four programs can be run simultaneously per computer. This figure has been modified from Sindhurakar et al., 2017, Neurorehabilitation and Neural Repair ${ }^{8}$. Please click here to view a larger version of this figure. 


\begin{tabular}{|c|c|c|c|}
\hline & Movement & Name/Definition & Solution \\
\hline 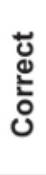 & & $\begin{array}{l}\qquad 2 \text { O'Clock Grasp } \\
\text { A grasp from " } 2 \text { O'Clock" or } \\
\text { higher on the knob that leads } \\
\text { to pronounced supination } \\
\text { behavior. }\end{array}$ & Not applicable. \\
\hline \multirow{2}{*}{ 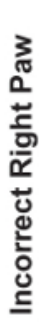 } & & $\begin{array}{l}\text { 3 O'Clock Grasp } \\
\text { A grasp from "3 O'Clock" on } \\
\text { the knob manipulandum that } \\
\text { results in incorrect supination } \\
\text { behavior. }\end{array}$ & \multirow{2}{*}{$\begin{array}{l}\text { At first sighting, constrain } \\
\text { aperture near the bottom of } \\
\text { the manipulandum. This } \\
\text { forces the animal to adopt a } \\
\text { higher paw placement. }\end{array}$} \\
\hline & & $\begin{array}{l}6 \text { O'Clock Grasp } \\
\text { A grasp from " } 6 \text { O'Clock" on } \\
\text { the knob manipulandum that } \\
\text { results in a pulling motion. }\end{array}$ & \\
\hline \multirow{2}{*}{ 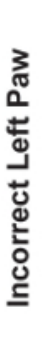 } & & $\begin{array}{l}\text { Interference } \\
\text { An animal extends the left paw } \\
\text { through the aperture, shifting } \\
\text { trunk posture and position to } \\
\text { aid in supination motion. }\end{array}$ & $\begin{array}{l}\text { Constrain the top portion of } \\
\text { the aperture to prevent left } \\
\text { paw reach. }\end{array}$ \\
\hline & & $\begin{array}{l}\text { Steering Wheel } \\
\text { Animals grasp with both paws } \\
\text { and attempt to supinate, using } \\
\text { a steering motion. }\end{array}$ & $\begin{array}{l}\text { Use constraint for left paw } \\
\text { interference, and manually } \\
\text { feed the animal when it uses } \\
\text { the appropriate paw to } \\
\text { maintain motivation. This } \\
\text { shapes out left paw reach. }\end{array}$ \\
\hline
\end{tabular}

Figure 2: Supination Movements. Diagrams and descriptions of common correct and incorrect supination movements encountered during the training protocol. Correct movements allow for true supination, while incorrect movements include compensatory mechanisms that may prevent true supination. Included are suggested solutions to correct improper movements. Please click here to view a larger version of this figure. 


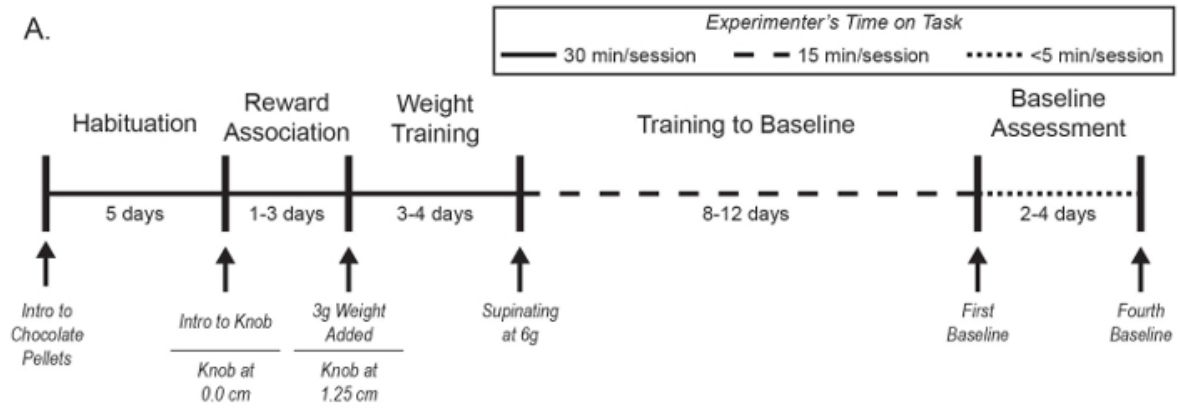

B.

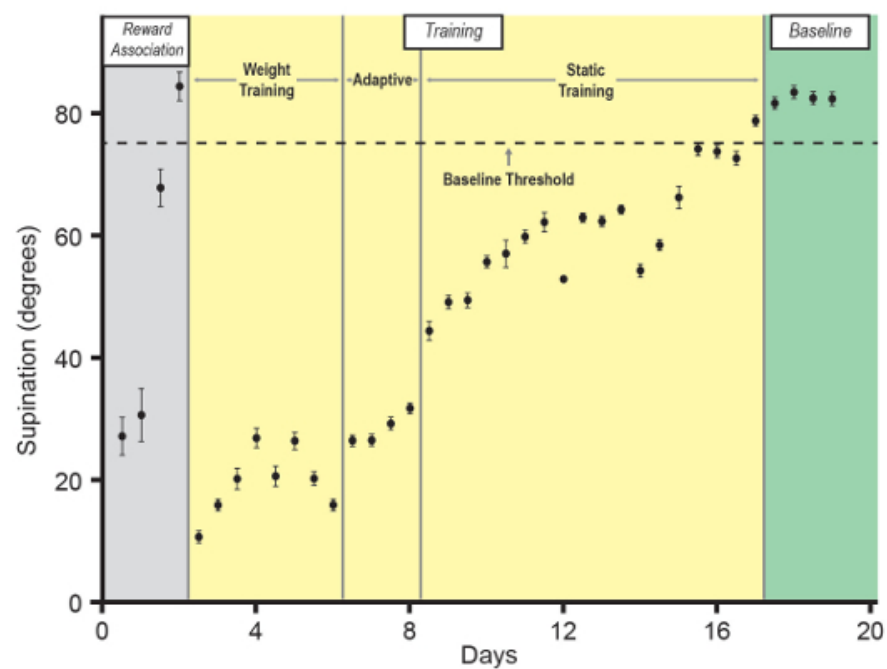

Figure 3: Training protocol. (A) Standard timeline. There are five periods of training lasting approximately 25 days in total: Habituation (5 d), Reward Association (1 - 3d), Weight Training (3 - 4 d), Training to Baseline (8 - 12 d), and Baseline Assessment (2 - 4 d). The line pattern on the timeline designates the time required of the experimenter to spend on the task each session. As the training protocol proceeds, the time on task decreases. (B) General progression of a rat's ability to supinate from reward association to baseline assessment. Overall, there is a positive linear progression of the rat towards baseline, but as observed, there is variability in a rat's performance throughout the training protocol. After weight training, there is a period of adaptive training, where the supination angle threshold is changed to match the rat's performance. This adaptive training is followed by a static thresholding paradigm until the rat has achieved baseline. Please click here to view a larger version of this figure. 
A.

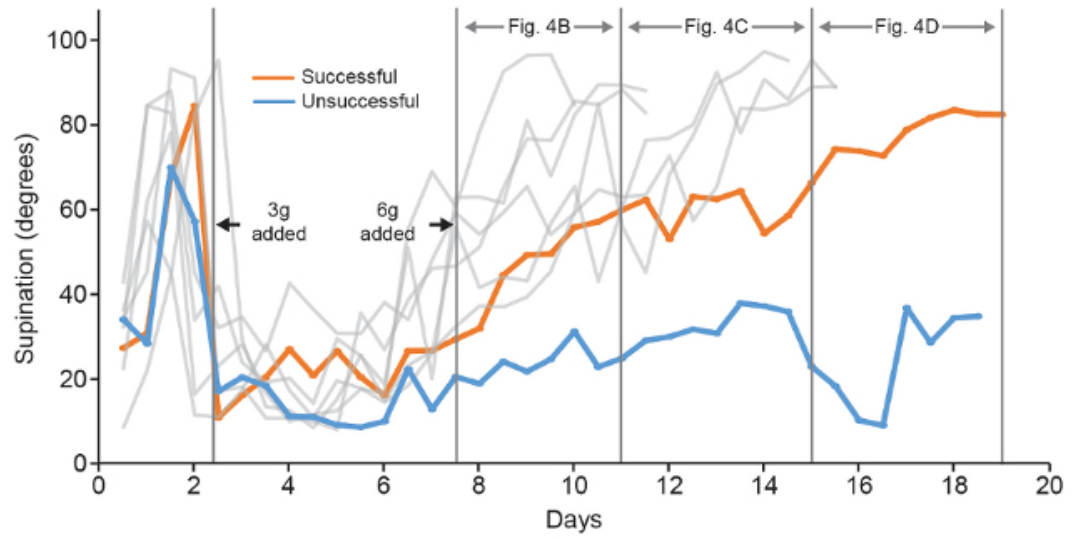

B.

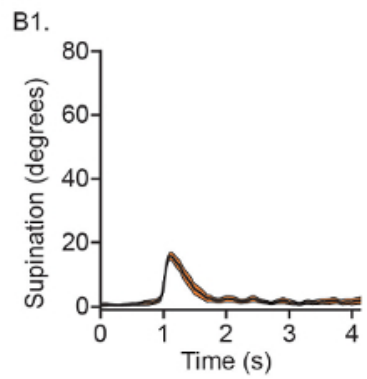

B2.

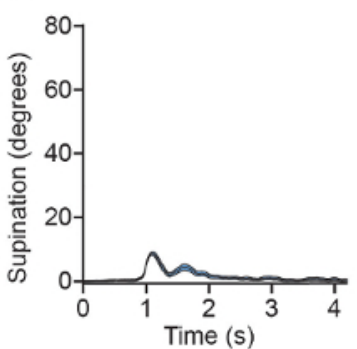

c.

C1.

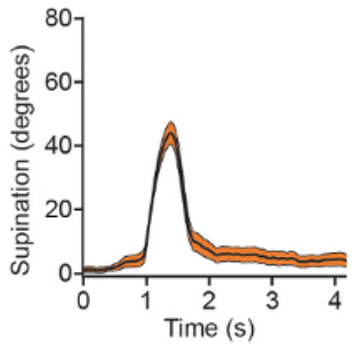

C2.

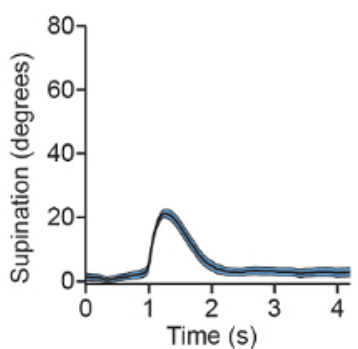

D.

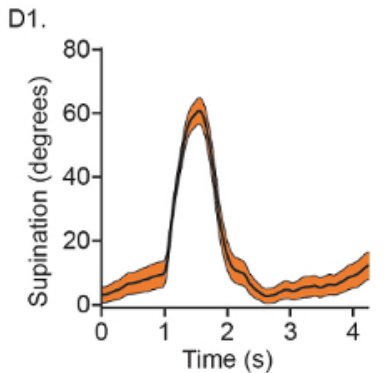

D2.

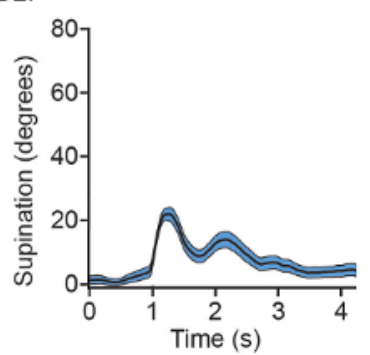

Figure 4: Successful and Unsuccessful Task Acquisition. (A) Progression of supination angle throughout training protocol for eight rats, seven successful and one unsuccessful. One representative rat that reaches baseline criterion (successful, orange) and one unsuccessful rat (blue) are further used as case studies. In the first seven days of the training after habituation, both the successful and unsuccessful rat showed similar progress in supination angle. By Day 11 post-habituation, the successful rat was supinating $55^{\circ}$ while the unsuccessful rat supinated $25^{\circ}$. After Day 15 post-habituation, the successful rat showed a strong upward progression, while the unsuccessful rat declined in performance. In the final third of training post-habituation, the unsuccessful rat had plateaued at $30^{\circ}$ while the successful rat was supinating $80^{\circ}$. (B) Average waveform (black line) with a 95\% confidence interval (orange for successful, blue for unsuccessful) for the first third of training after $6 \mathrm{~g}$ of counterweight is added. (B1) Successful rat - single peak around $20^{\circ}$. (B2) Unsuccessful rat - double peak with global maximum of $10^{\circ}$. (C) Average waveform (black line) with a $95 \%$ confidence interval (orange for successful, blue for unsuccessful) for the second third of training after $6 \mathrm{~g}$ of counterweight is added. (C1) Successful rat - single peak at $45^{\circ}$. (C2) Unsuccessful rat - improved form with single peak near $20^{\circ}$. (D) Average waveform (black line) with a $95 \%$ confidence interval (orange for successful, blue for unsuccessful) for the final third of training after 6 $\mathrm{g}$ of counterweight is added. (D1) Successful rat - pronounced single peak at $65^{\circ}$. (D2) Unsuccessful rat - double peak with global maximum at $20^{\circ}$. Please click here to view a larger version of this figure. 
A.

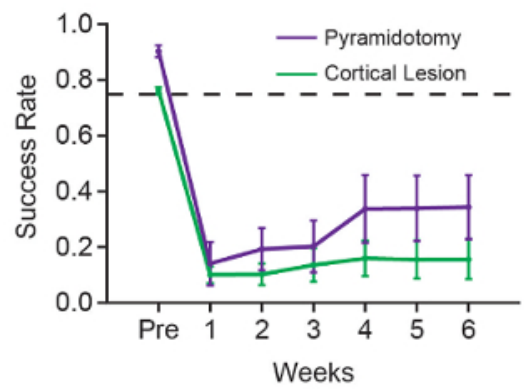

B.

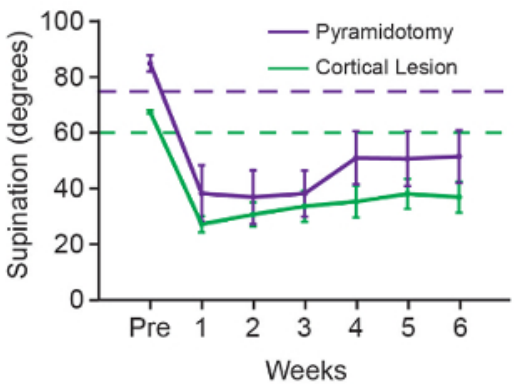

Figure 5: Task Sensitivity to Different Injury Models. Rats in the pyramidotomy group (purple, $\mathrm{n}=8$ ) were trained to supinate $75^{\circ}$ at $6 \mathrm{~g}$ at a success rate of $75 \%$ or above, while rats in the cortical lesion group (green, $\mathrm{n}=10$ ) were trained to supinate $60^{\circ}$ at $6 \mathrm{~g}$ at $75 \%$ or above. Data shown are mean \pm standard error. (A) Success rate for pyramidotomy lesion versus cortical lesion. Both injury models showed a sharp decrease in success rate from pre- to post-injury (week 1). Success rate for pyramidotomy decreased from $0.90 \pm 0.02$ to $0.14 \pm 0.08$, while success rate for cortical lesion decreased from $0.76 \pm 0.01$. (B) Supination angle for pyramidotomy versus cortical lesion. Both groups showed a decrease from pre- to post-injury: the pyramidotomy group decreased to $38.2^{\circ} \pm 10.1^{\circ}$ while the cortical lesion group decreased to $27.1^{\circ} \pm 2.9^{\circ}$. Please click here to view a larger version of this figure.

\section{Discussion}

The knob supination task evaluates forelimb supination in rats using quantitative and semi-automated methods. To achieve these endpoints, many of the parameters designed for the task, including knob alignment, manipulandum design, and training criteria, have been iterated over several years. For knob alignment, we experimented with three different alignments of the knob with regards to the aperture: left side of the knob aligned with the left side of the aperture, the knob centered in aperture, and the right side of the knob aligned with the right side of the aperture. We settled on the right side of the knob being aligned with the right side of the aperture, as this produced rats that were trained in the shortest amount of time and who supinated with minimal compensatory mechanisms, specifically, interference from the left paw.

As for manipulandum design, we altered several design features to maximize turning with the forelimb and minimize use of the body. In addition, we scaled the difficulty of the task to the severity of the predicted deficit. After pyramidotomy, supination is the movement that is most strongly affected, but the impairment is still relatively subtle. Thus, we trained the rats to a higher baseline criterion $\left(75^{\circ}\right)$ to ensure that large deficits were observed after injury. For cortical lesions, which are more impairing, 60-degree threshold at $7.5 \mathrm{~g}$ was sufficient to demonstrate a significant deficit after injury. Additional parameters that were optimized through a trial-and-error approach include aperture size, knob distance from aperture, and time window to achieve a successful trial.

There are some critical points throughout the training protocol that require careful supervision. When training to baseline, the adaptive thresholding method has been used successfully to train rats to $75^{\circ} 10$. However, rats can plateau at a peak angle less than $75^{\circ}$; the performance remains the same after 4-5 sessions. To improve performance, a static threshold can be employed. A static threshold refers to the threshold remaining at a set degree, which is independent of rat performance, as opposed to an adaptive threshold that changes based on recent performance. If the rat plateaus during adaptive training, the experimenter should change to a static threshold. Static training stages range from 20 to 70 degrees in $10^{\circ}$ increments. (Stage K28 - K33). Choose the static stage based on the rat's average peak angle in the previous 2 sessions. For example, if the rat is averaging $45^{\circ}$, select the static stage for 50 degrees (K31). All static stages set the "Init. Thresh." at $5^{\circ}$. During training, if the rat loses motivation, manually feed the rat if it supinates close to but not above the threshold.

In addition, during baseline assessment, approximately $5 \%$ of rats regress $5-10^{\circ}$ in their supination angle and $5-10 \%$ in success rate between sessions. If this happens, and the rat does not recover the $75^{\circ}$ average peak angle after $3-4$ sessions, decrease the static stage to within 10 degrees of the rat's current average angle before returning to step 3.5. It is important to not reintroduce a rat to the adaptive stages once it has been placed on static training stages.

There are some limitations to the task. Once incorrect grasp position has been established, changing grasping behavior (Figure 2) can be difficult. Thus, early detection and correction is important. To correct a rat's grasp, the aperture can be altered by narrowing the size of the aperture in the horizontal and/or vertical direction; typically we tape a glass slide to the edge of the aperture that needs adjustment. For most rats, this improves their grasp form because it forces them to grasp the manipulandum in a specific manner. This, in turn, improves their ability to properly supinate.

In addition to this challenge, rats can develop compensatory mechanisms to supinate. These include the use of the head to aid the forelimb in supination; lowering the elbow and shoulder joint to turn the knob; using the left paw to help turn the knob or push the reaching paw down. All of these behaviors can be used to successfully complete the task. As mentioned above, behaviors relating to the grasp can be corrected by manipulating the aperture. Compensatory mechanisms outside of the grasp, however, require active participation by the experimenter to not reward compensatory behavior. After injury, we have observed rats taking several trials to place the paw in the proper position before supinating. Although we have not analyzed which components of the task might contribute to the loss of supination, these could include loss of accurate grip and impaired force modulation, among many possibilities.

The semi-automated supination task takes, on average, $20 \pm 5$ days to train rats to baseline, and $25 \%$ of animals are unable to be trained on the task. Contributing to the training time is the fact that we have not selected naturally right-preference rats but instead force all animals to use their right paws as is common in most reaching assays. We have not tried using left-preference rats, but it would be an interesting exploratory study to 
first identify paw preference and then train the dominant paw. To accommodate this, we would need to flip the orientation of the doors so that the aperture is reversed; this can easily be done.

Compared with traditional tasks like the IBB or single pellet reaching, the supination task quantitatively and objectively measures forelimb reaching. It shows sensitivity to severe injury (cortical lesion) and subtle injury (pyramidotomy), and the training procedure can be modified depending on the severity of the injury model. Because it is semi-automated, the task allows the experimenter to train multiple rats simultaneously, depending on training stage. This greatly improves the experimenter's productivity and rat throughput. The task is reliable and reproducible between rats. By creating a troubleshooting guide (Figure 2) for experimenters to refer to during the training protocol, we have standardized several incorrect behaviors as well as solutions to fix them. Lastly, the task offers an intuitive means to analyze large amounts of data and gives the experimenter the ability to delve deeper into the kinetics of supination.

In the future, we will be using the semi-automated supination task as a platform to evaluate type, dose, and timing of rehabilitation. Our lab is interested in the effects of stimulation on functional improvement after injury. In addition, we are interested in how therapies which stimulate neural repair or improve neural conduction and communication can affect rehabilitation. We also have interest in modifying the task to be compatible with electrophysiology so that we can study motor learning; rats with head caps routinely perform the task, and adding a commutator for recording or stimulation would be simple to do. The task, as described, is for rats, but there are also labs experimenting with using mice for the task. In general, this task can be used for evaluating forelimb function in rodents in a wide variety of injury models and disease states and in turn, for evaluating rehabilitative strategies. Moving forward, we will continue to improve the task, with refinements to help lessen incorrect behaviors and improve task acquisition rate and training time.

\section{Disclosures}

Dr. Rennaker and Dr. Sloan are the owners of Vulintus Inc., which manufactures the equipment in this publication. No conflicts of interest declared for the other authors.

\section{Acknowledgements}

This research has been funded by NIH-NINDS R03 NS091737.

\section{References}

1. Anderson, K. D. Targeting recovery: priorities of the spinal cord-injured population. J Neurotraum. 21 (10), 1371-1383 (2004).

2. Martin, J. H. Systems neurobiology of restorative neurology and future directions for repair of the damaged motor systems. Clin Neurol Neurosur. 114 (5), 515-523 (2012).

3. Lemon, R. N. Descending pathways in motor control. Annu Rev Neurosci. 31, 195-218 (2008).

4. Feng, W., Wang, J., et al. Corticospinal tract lesion load: An imaging biomarker for stroke motor outcomes. Ann Neurol. 78 (6), 860-870 (2015).

5. Whishaw, I. Q., Pellis, S. M., Gorny, B. P., \& Pellis, V. C. The impairments in reaching and the movements of compensation in rats with motor cortex lesions: an endpoint, videorecording, and movement notation analysis. Behav Brain Res. 42 (1), 77-91 (1991).

6. Irvine, K.-A., Ferguson, A. R., Mitchell, K. D., Beattie, S. B., Beattie, M. S., \& Bresnahan, J. C. A novel method for assessing proximal and distal forelimb function in the rat: the Irvine, Beatties and Bresnahan (IBB) forelimb scale. J Vis Exp. (46) (2010).

7. Allred, R. P., Adkins, D. L., et al. The vermicelli handling test: a simple quantitative measure of dexterous forepaw function in rats. $J$ Neurosci Meth. 170 (2), 229-244 (2008).

8. Sindhurakar, A., Butensky, S. D., et al. An automated test of rat forelimb supination quantifies motor function loss and recovery after corticospinal injury. Neurorehab Neural Re. 31 (2), 122-132 (2017).

9. Carmel, J. B., Kim, S., Brus-Ramer, M., \& Martin, J. H. Feed-forward control of preshaping in the rat is mediated by the corticospinal tract. Eur J Neurosci. 32 (10), 1678-1685 (2010).

10. Meyers, E., Sindhurakar, A., et al. The supination assessment task: An automated method for quantifying forelimb rotational function in rats. $J$ Neurosci Meth. 266, 11-20 (2016).

11. Gomez-Marin, A., Paton, J. J., Kampff, A. R., Costa, R. M., \& Mainen, Z. F. Big behavioral data: psychology, ethology and the foundations of neuroscience. Nat Neurosci. 17 (11), 1455-1462 (2014)

12. Wong, C. C., Ramanathan, D. S., Gulati, T., Won, S. J., \& Ganguly, K. An automated behavioral box to assess forelimb function in rats. J Neurosci Meth. 246, 30-37 (2015).

13. Alaverdashvili, M., \& Whishaw, I. Q. Motor cortex stroke impairs individual digit movement in skilled reaching by the rat. Eur J Neurosci. 28 (2), 311-322 (2008).

14. Ellens, D. J., Gaidica, M., et al. An automated rat single pellet reaching system with high-speed video capture. J Neurosci Meth. 271, 119-127 (2016).

15. Lai, S., Panarese, A., et al. Quantitative kinematic characterization of reaching impairments in mice after a stroke. Neurorehab Neural Re. 29 (4), 382-392 (2015).

16. Molina-Luna, K., Hertler, B., Buitrago, M. M., \& Luft, A. R. Motor learning transiently changes cortical somatotopy. Neuroimage. 40 (4), 1748-1754 (2008).

17. Ballermann, M., Tompkins, G., \& Whishaw, I. Q. Skilled forelimb reaching for pasta guided by tactile input in the rat as measured by accuracy, spatial adjustments, and force. Behav Brain Res. 109 (1), 49-57 (2000).

18. Vigaru, B. C., Lambercy, O., et al. A robotic platform to assess, guide and perturb rat forelimb movements. IEEE Trans Neural Syst Rehabil Eng. 21 (5), 796-805 (2013).

19. Martin, J. H., Choy, M., Pullman, S., \& Meng, Z. Corticospinal system development depends on motor experience. J Neurosci. 24 (9), 2122-2132 (2004). 
20. Murgia, A., Kyberd, P. J., Chappell, P. H., \& Light, C. M. Marker placement to describe the wrist movements during activities of daily living in cyclical tasks. Clin Biomech. 19 (3), 248-254 (2004).

21. Simpson, J., \& Kelly, J. P. An investigation of whether there are sex differences in certain behavioural and neurochemical parameters in the rat. Behav Brain Res. 229 (1), 289-300 (2012).

22. Hays, S. A., Khodaparast, N., et al. The isometric pull task: a novel automated method for quantifying forelimb force generation in rats. $J$ Neurosci Meth. 212 (2), 329-337 (2013). 